\title{
On MSE-Optimal Circular Crossover Designs
}

\author{
C. Neumann ${ }^{1} \cdot$ J. Kunert ${ }^{1}$
}

Accepted: 30 July 2021 / Published online: 12 November 2021

(C) The Author(s) 2021

\begin{abstract}
In crossover designs, each subject receives a series of treatments, one after the other in $p$ consecutive periods. There is concern that the measurement of a subject at a given period might be influenced not only by the direct effect of the current treatment but also by a carryover effect of the treatment applied in the preceding period. Sometimes, the periods of a crossover design are arranged in a circular structure. Before the first period of the experiment itself, there is a run-in period, in which each subject receives the treatment it will receive again in the last period. No measurements are taken during the run-in period. We consider the estimate for direct effects of treatments which is not corrected for carryover effects. If there are carryover effects, this uncorrected estimate will be biased. In that situation, the quality of the estimate can be measured by the mean square error, the sum of the squared bias and the variance. We determine MSE-optimal designs, that is, designs for which the mean square error is as small as possible. Since the optimal design will in general depend on the size of the carryover effects, we also determine the efficiency of some designs compared to the locally optimal design. It turns out that circular neighbour-balanced designs are highly efficient.
\end{abstract}

Keywords Optimal design · Crossover design · Carryover effect · MSE-optimality

Mathematics Subject Classification $62 \mathrm{~K} 05 \cdot 62 \mathrm{~K} 10$

This article is part of the topical collection "Special Issue: State of the art in research on design and analysis of experiments" guest edited by John Stufken, Abhyuday Mandal, and Rakhi Singh.

\footnotetext{
$凶 \quad$ J. Kunert

joachim.kunert@tu-dortmund.de

C. Neumann

christoph2.neumann@tu-dortmund.de

1 Department of Statistics, TU Dortmund University, 44221 Dortmund, Germany
} 


\section{Introduction}

In a crossover design, the effects of $t$ treatments are compared by giving each of $n$ subjects a series of treatments in $p$ consecutive periods. The response of each unit in each period is measured and used to estimate the so-called direct effects of the treatments. Usually, for experiments of this kind, there is concern that a treatment given in period $i, 1 \leq i \leq p$, in addition to its direct effect will also have a carryover effect that influences the measurement in period $i+1$. An important application of crossover designs is in sensory experiments, where each assessor evaluates a number of products, one after the other. Here, a carryover effect may for instance be caused by a lingering taste of a product.

There are two options to deal with carryover effects. One possibility is to explicitly include carryover effects in the model and use an estimate that corrects for carryover effects. There is a vast literature on designs for the corrected estimate, see, e.g. [3].

Another approach is to neglect the carryover effect and use an uncorrected estimate which does not correct for carryover effects. In sensory experiments, the analysis is usually done with the uncorrected estimate. In most cases, the experimenter then uses technical measures like, e.g. washout with a neutral taste to reduce carryover.

To deal with the, hopefully small, carryover effects which may be present in spite of washout, some authors recommend using neighbour-balanced designs as a precaution to minimize the effects of carryover, see, e.g. [11, p. 189]. Neumann and Kunert [12] have formally shown that the use of neighbour-balanced designs can minimize the mean square error of the uncorrected estimate.

In a circular crossover design, there is a run-in period where each unit receives a treatment but no measurement is made. During the run-in period of a circular design, each unit receives the treatment which it will receive again in the last period. Hence, there is a carryover effect in each period. Circular designs were introduced to the area of crossover designs by Magda [10]. There are some results on optimal designs for estimators taking account of the carryover effects, e.g. by Kunert [7], Druilhet [5] and Bailey and Cameron [2].

Azaiis [1] has shown that for circular designs and the uncorrected estimate, there is a randomization which preserves the neighbour structure but validates an analysis with the simple block model. This makes circular designs appealing for the uncorrected estimate.

The present paper extends the results of [12] to the class of circular designs. Since the mean square error depends on the size of the carryover effects, we determine locally optimal designs. If the number of periods is larger than the number of treatments, however, it turns out that there are designs minimizing the mean square error regardless of the size of the carryover effects. If the number of periods is less or equal to the number of treatments, the optimal design varies with the size of the carryover effects. As long as the carryover effects are small, no experimental unit in the optimal design receives the same treatment twice. When the carryover effects get larger, some units will receive the same treatment twice in consecutive periods. The proportion of units receiving the same treatment twice increases with increasing carryover effects. These results remain correct whether there is a period effect in the model or not. 


\section{Calculating the MSE}

Define $\Omega_{t, n, p}$ as the set of all circular crossover designs with $t$ treatments, $n$ units (subjects) and $p$ periods. We assume that the $j$-th observation on unit $i$ can be written as

$$
y_{i j}=\alpha_{i}+\tau_{d(i, j)}+\rho_{d(i, j-1)}+\varepsilon_{i j}
$$

Here, $\alpha_{i}, 1 \leq i \leq n$, is the effect of the $i$-th unit, $\tau_{d(i, j)}$ is the effect of the treatment given to the $i$-th unit in the $j$-th period by the design $d \in \Omega_{t, n, p}, \rho_{d(i, j-1)}$ is the carryover effect of the treatment given to unit $i$ in period $(j-1)$ with $\rho_{d(i, 0)}=\rho_{d(i, p)}$, and $\varepsilon_{i j}$ is the error. The errors are independent, identically distributed with expectation 0 and variance $\sigma^{2}$.

To continue, we need some notation. Denote by $A^{T}$ the transpose of the matrix $A$. If $I_{t}$ is the $t \times t$-identity matrix and $1_{t}$ the $t$-dimensional vector of ones, define $H_{t}=I_{t}-\frac{1}{t} 1_{t} 1_{t}^{T}$. For a matrix $A \in \mathbb{R}^{(n, r)}$ set $\omega^{\perp}(A)=I_{n}-A\left(A^{T} A\right)^{+} A^{T}$, where $B^{+}$denotes the Moore-Penrose generalized inverse of the matrix $B$.

In vector notation, our model then can be written as

$$
y=U \alpha+T_{d} \tau+F_{d} \rho+\varepsilon .
$$

Here, $y=\left[y_{11}, \ldots, y_{1 p}, y_{21}, \ldots, y_{n p}\right]^{T}$ and $\varepsilon$ is the vector of the errors. Further, $\alpha, \tau$ and $\rho$ are the vectors of the unit, direct and carryover effects, respectively. The matrices $U, T_{d}$ and $F_{d}$ are the corresponding design matrices.

We assume that the analysis of the data is done with a model neglecting the carryover effects, i.e.

$$
y=U \alpha+T_{d} \tau+\varepsilon
$$

If we define $M_{d 11}=T_{d}^{T} \omega^{\perp}(U) T_{d}$, then $M_{d 11}$ is the information matrix for the estimation of direct effects in model (2). Note that $M_{d 11}$ and, therefore, $M_{d 11}^{+}$have rowand column-sums 0 . Consequently, $H_{t} M_{d 11}^{+}=M_{d 11}^{+}$and $\hat{\tau}=M_{d 11}^{+} T_{d}^{T} \omega^{\perp}(U) y$ is the Gauss-Markov estimate for $H_{t} \tau$ in model (2).

If there are carryover effects, then the uncorrected estimate $\hat{\tau}$ is biased. More precisely,

$$
E(\hat{\tau})=H_{t} \tau+M_{d 11}^{+} T_{d}^{T} \omega^{\perp}(U) F_{d} \rho
$$

As in [12], we try to determine a design that minimizes the mean square error (MSE) as a performance measure combining bias and variance.

The joint information matrix of direct and carryover effects in model (1) can be written as

$$
M_{d}=\left[\begin{array}{ll}
M_{d 11} & M_{d 12} \\
M_{d 12}^{T} & M_{d 22}
\end{array}\right]
$$


where $M_{d 11}$ is as before and

$$
\begin{aligned}
& M_{d 12}=T_{d}^{T} \omega^{\perp}(U) F_{d}, \\
& M_{d 22}=F_{d}^{T} \omega^{\perp}(U) F_{d},
\end{aligned}
$$

see [3, p. 15].

Analogous to [12], we calculate the MSE in the following way: We restrict attention to designs which allow estimation of all contrasts of direct effects in the model without carryover effects. Because $M_{d 11}$ is the information matrix for direct effects in the model without carryover effects, this is the set of all designs for which $\operatorname{rank}\left(M_{d 11}\right)=t-1$. In the model with carryover effects, the MSE of the uncorrected estimate $\widehat{\tau_{i}-\tau_{j}}$ for any pair $(i, j), i \neq j$ then is

$$
E\left(\widehat{\tau_{i}-\tau_{j}}-\left(\tau_{i}-\tau_{j}\right)\right)^{2}=\sigma^{2} \ell_{i j}^{T} M_{d 11}^{+} \ell_{i j}+\left(\ell_{i j}^{T} M_{d 11}^{+} M_{d 12} \rho\right)^{2}
$$

where $\ell_{i j}$ is a $t$-dimensional vector with +1 in position $i,-1$ in position $j$ and all other entries 0 . With the calculations done in [12], we get that the average MSE over all pairs equals

$$
\frac{2}{t-1}\left(\sigma^{2} \operatorname{tr}\left(M_{d 11}^{+}\right)+\rho^{T}\left(M_{d 12}^{T} M_{d 11}^{+} M_{d 11}^{+} M_{d 12}\right) \rho\right)
$$

where $\operatorname{tr}(M)$ denotes the trace of a matrix $M$. As in [12], we consider the worst case for given $\rho^{T} H_{t} \rho=\sum\left(\rho_{i}-\bar{\rho}\right)^{2}=\delta$, say. That is, we consider

$$
\begin{aligned}
& \max _{\rho^{T} H_{t} \rho=\delta}\left(\frac{2}{t-1}\left(\sigma^{2} \operatorname{tr}\left(M_{d 11}^{+}\right)+\rho^{T}\left(M_{d 12}^{T} M_{d 11}^{+} M_{d 11}^{+} M_{d 12}\right) \rho\right)\right) \\
& =\frac{2}{t-1}\left(\sigma^{2} \operatorname{tr}\left(M_{d 11}^{+}\right)+\delta \lambda_{1}\left(M_{d 12}^{T} M_{d 11}^{+} M_{d 11}^{+} M_{d 12}\right)\right),
\end{aligned}
$$

where $\lambda_{i}(M)$ is the $i$-th largest eigenvalue of the symmetric matrix $M$.

We multiply this equation by $\frac{t-1}{2}$ and get the following definition for our optimalitycriterion. For any $d \in \Omega_{t, n, p}$, we define

$$
\operatorname{MSE}(d)=\sigma^{2} \operatorname{tr}\left(M_{d 11}^{+}\right)+\delta \lambda_{1}\left(M_{d 12}^{T} M_{d 11}^{+} M_{d 11}^{+} M_{d 12}\right)
$$

The advantage of this criterion is that the multivariate purpose of minimizing the bias and maximizing the precision of the estimators can be measured as a single real number. In what follows, we assume without loss of generality that $\sigma^{2}=1$. Our aim is to find a design that minimizes $\operatorname{MSE}(d)$.

We call a square matrix $M$ completely symmetric, if there are numbers $a$ and $b$ such that all diagonal elements are equal to $a$ and all off-diagonal elements are equal to $b$. We get the following bound for $\operatorname{MSE}(d)$. 
Theorem 1 For any design $d \in \Omega_{t, n, p}$, there is a lower bound for $\operatorname{MSE}(d)$, namely

$$
\operatorname{MSE}(d) \geq \frac{(t-1)^{2}}{\operatorname{tr}\left(M_{d 11}\right)}+\delta \frac{\left(\operatorname{tr}\left(M_{d 12}\right)\right)^{2}}{\left(\operatorname{tr}\left(M_{d 11}\right)\right)^{2}} .
$$

Equality holds if both $M_{d 11}$ and $M_{d 12}$ are completely symmetric.

Proof The proof given for Proposition 1 of [12] remains valid in the circular case.

Note that for every $M_{d 11}$ and $M_{d 12}$ there are symmetrized versions $\bar{M}_{d 11}$ and $\bar{M}_{d 12}$ with

$$
\bar{M}_{d i j}=\frac{1}{t !} \sum_{\Pi \in \mathcal{S}_{t}} \Pi^{T} M_{d i j} \Pi
$$

for $1 \leq i \leq j \leq 2$. Here $\mathcal{S}_{t}$ is the set of all $(t \times t)$-permutation matrices $\Pi$. We see that all $\bar{M}_{d i j}$ are completely symmetric and that $\operatorname{tr}\left(M_{d i j}\right)=\operatorname{tr}\left(\bar{M}_{d i j}\right)$.

For any design $d$, define $q_{d i j}=\frac{1}{n} \operatorname{tr}\left(M_{d i j}\right)$ for $1 \leq i \leq j \leq 2$. Then, the lower bound in Theorem 1 can be written as

$$
\operatorname{MSE}(d) \geq \frac{(t-1)^{2}}{n q_{d 11}}+\delta\left(\frac{q_{d 12}}{q_{d 11}}\right)^{2} .
$$

Each subject in the design $d$ receives a sequence $s$ of treatments. Denote by $T(s)$ and $F(s)$ the part of $T_{d}$ and $F_{d}$ that corresponds to $s$ and define

$$
q_{11}(s)=\operatorname{tr}\left(T(s)^{T} \omega^{\perp}\left(1_{p}\right) T(s)\right) \quad \text { and } \quad q_{12}(s)=\operatorname{tr}\left(T(s)^{T} \omega^{\perp}\left(1_{p}\right) F(s)\right) .
$$

We call two sequences $s_{1}$ and $s_{2}$ equivalent if $s_{1}$ can be transformed to $s_{2}$ by relabelling the treatments. Two equivalent sequences then have the same $q_{i j}(s)$. If for given $t$ and $p$ there are $K$, say, equivalence classes of sequences, we choose a representative sequence $s_{k}, 1 \leq k \leq K$ for each class. As pointed out by Kushner [9], the $q_{d i j}$ then are weighted means of the $q_{i j}\left(s_{k}\right)$. More precisely, we get

$$
q_{d i j}=\sum_{k=1}^{K} q_{i j}\left(s_{k}\right) \pi_{d}(k),
$$

where $\pi_{d}(k)$ is the proportion of units of $d$ receiving a sequence from class $k, 1 \leq$ $k \leq K$.

Similarly to [12], we consider approximate designs. For approximate designs, we remove the restriction that the number of experimental units to receive a given sequence $s$ must be an integer. For an approximate design, the $\pi_{d}(k), 1 \leq k \leq K$ can be any set of nonnegative real numbers, subject to the condition that $\sum_{k=1}^{K} \pi_{d}(k)=1$. An exact design $d \in \Omega_{t, n, p}$ then is a special instance of an approximate design, where each sequence is assigned to an integral number of units. We denote the set of all 
Table 1 Size of $q_{11}(s)$ and $q_{12}(s)$ for two classes of sequences in the case $p>t$

\begin{tabular}{llll}
\hline Class & Sequence & $q_{11}(s)$ & $q_{12}(s)$ \\
\hline$A$ & $s_{1}$ & $p-\left(g^{2}(t-s)-(g+1)^{2} s\right) / p$ & $p-t-\left(g^{2}(t-s)-(g+1)^{2} s\right) / p$ \\
$B$ & $s_{2}$ & $p-\left(g^{2}(t-s)-(g+1)^{2} s\right) / p$ & $-\left(g^{2}(t-s)-(g+1)^{2} s\right) / p$ \\
\hline
\end{tabular}

approximate designs for given $t, n$ and $p$ by $\Delta_{t, n, p}$. Note that the number $n$ of units is not important for an approximate design $d \in \Delta_{t, n, p}$. It plays a role in the calculation of $\operatorname{MSE}(d)$, however. A design $d \in \Delta_{t, n, p}$ is called symmetric, if each sequence $s$ from class $k, 1 \leq k \leq K$ appears equally often (i.e. $\pi_{d}(k) / m_{k}$ times, where $m_{k}$ is the number of sequences in class $k$ ). If the design $d$ is symmetric, then all $M_{d i j}$ are completely symmetric, $1 \leq i \leq j \leq 2$. We see from the definition of symmetrized designs that for every combination of $\operatorname{tr}\left(M_{d 11}\right)$ and $\operatorname{tr}\left(M_{d 12}\right)$ there is a symmetric design with the same traces. As these designs achieve the lower bound in Theorem 1, we can restrict attention to symmetric designs.

\section{Optimal Designs}

For any sequence $s$, we observe that

$$
q_{11}(s)=p-\frac{1}{p} \sum_{m=1}^{t} f_{s, m}^{2} \text { and } q_{12}(s)=B_{s}-\frac{1}{p} \sum_{m=1}^{t} f_{s, m}^{2} \text {, }
$$

where $f_{s, m}$ is the frequency of treatment $m$ in sequence $s$ and $B_{s}$ the number of periods where the treatment of the preceding period is repeated. These formulas show that the $q_{i j}(s)$ depend on the sequence only through the treatment frequencies and the number of periods where the preceding treatment is repeated. The position of the treatments within the sequence does not matter. Hence, we will choose the representative sequences such that the periods with repeated treatments, if there are, come at the end.

As a first step, let $p>t$. In this case, we can write $p=g t+s$ with $g$ and $s$ being integers and $1 \leq s \leq t$. In this situation, there are two classes of particular interest, namely $A$ with representative sequence $s_{1}$ and $B$ with representative sequence $s_{2}$, where

$$
\begin{aligned}
& s_{1}=[1, \ldots, 1,2, \ldots, 2, \ldots, t-1, \ldots, t-1, t, \ldots, t] \\
& s_{2}=[1,2, \ldots, t, 1,2, \ldots, t, \ldots, 1,2, \ldots, t, 1,2, \ldots, r]
\end{aligned}
$$

In these sequences, each treatment appears either $g$ or $g+1$ times. Note that it makes no difference for $q_{11}\left(s_{1}\right)$ and $q_{12}\left(s_{1}\right)$ whether the treatments appearing $g+1$ times come early or late in $s_{1}$. 
Table 2 Size of $q_{11}(s)$ and $q_{12}(s)$ for the two classes of possible sequences when $2=p \leq t$

\begin{tabular}{llll}
\hline Class & Sequence & $q_{11}(s)$ & $q_{12}(s)$ \\
\hline$A$ & {$[1,1]$} & 0 & 0 \\
$B$ & {$[1,2]$} & 1 & -1 \\
\hline
\end{tabular}

The values of $q_{11}(s)$ and $q_{12}(s)$ for the two classes $A$ and $B$ are displayed in Table 1. It can be seen that $\sum_{m=1}^{t} f_{s, m}^{2}$ is minimal, if the $f_{s, m}, 1 \leq m \leq t$, are as nearly equal as possible. Consequently, $s_{1}$ and $s_{2}$ maximize $q_{11}(s)$. With that observation, the MSEoptimal design can be determined as follows.

Theorem 2 Assume $p>t$ and consider a symmetric approximate design $d^{*} \in \Delta_{t, n, p}$ consisting of sequences from class A with proportion

$$
\pi_{1}=\frac{p^{2}-s^{2}+t s}{p t(p-t)}
$$

and of sequences from class $B$ with proportion $\pi_{2}=1-\pi_{1}$. We then get

$$
\forall d \in \Delta_{t, n, p}: \operatorname{MSE}(d) \geq \operatorname{MSE}\left(d^{*}\right)
$$

Proof In each sequence used by $d^{*}$, each treatment in each unit appears either $g$ or $g+1$ times. Therefore, we have that

$$
q_{d}^{*} 11=\max _{d}\left\{q_{d 11}\right\}
$$

and

$$
\operatorname{MSE}(d) \geq \frac{(t-1)^{2}}{n q_{d 11}}+\delta\left(\frac{q_{d 12}}{q_{d 11}}\right)^{2} \geq \frac{(t-1)^{2}}{n q_{d 11}} \geq \frac{(t-1)^{2}}{n q_{d^{*} 11}}
$$

Observe that the choice of $\pi_{1}$ implies that $q_{d^{*} 12}=0$. Since $d^{*}$ is symmetric, we get

$$
\operatorname{MSE}\left(d^{*}\right)=\frac{(t-1)^{2}}{n q_{d^{*} 11}},
$$

which completes the proof.

If $p \leq t$, the determination of an MSE-optimal design is more complicated. We examine the special cases $p=2 \leq t$ and $p=3 \leq t$ before we find general optimal designs for $4 \leq p \leq t$.

Assume $p=2$. Note that there are only two possible classes of sequences $A$ and $B$, displayed in Table 2 . 
Table 3 Size of $q_{11}(s)$ and $q_{12}(s)$ for the five classes of possible sequences when $3=p \leq t$

\begin{tabular}{llll}
\hline Class & Sequence & $q_{11}(s)$ & $q_{12}(s)$ \\
\hline$A$ & {$[1,1,1]$} & 0 & 0 \\
$B_{1}$ & {$[1,2,2]$} & $4 / 3$ & $-2 / 3$ \\
$B_{2}$ & {$[1,1,2]$} & $4 / 3$ & $-2 / 3$ \\
$B_{3}$ & {$[1,2,1]$} & $4 / 3$ & $-2 / 3$ \\
$C$ & {$[1,2,3]$} & 2 & -1 \\
\hline
\end{tabular}

Theorem 3 Assume $2=p \leq t$ and consider a symmetric approximate design $d^{*} \in$ $\Delta_{t, n, 2}$ consisting only of sequences from class $B$ with representative sequence $[1,2]$. Then,

$$
\forall d \in \Delta_{t, n, 2}: \operatorname{MSE}(d) \geq \operatorname{MSE}\left(d^{*}\right)
$$

Proof Assume the design has proportion $\pi_{1}$ of its sequences from class $A$ and $\pi_{2}=$ $1-\pi_{1}$ from class $B$. We get from Table 2 that $q_{d 11}=\sum \pi_{d}(s) q_{11}(s)=\pi_{2}$ and $q_{d 12}=-\pi_{2}$. Hence,

$$
\operatorname{MSE}(d) \geq \frac{(t-1)^{2}}{n \pi_{2}}+\delta\left(\frac{-\pi_{2}}{\pi_{2}}\right)^{2}=\frac{(t-1)^{2}}{n \pi_{2}}+\delta \geq \frac{(t-1)^{2}}{n}+\delta=\operatorname{MSE}\left(d^{*}\right) .
$$

This completes the proof.

For the case $p=3$, there are only the five possible classes of sequences listed in Table 3. In this case, the optimal design consists of sequences [1, 2, 3] only.

Theorem 4 Assume $3=p \leq t$ and consider a symmetric approximate design $d^{*} \in \Delta_{t, n, 3}$ that consists only of sequences from class $C$ with representative sequence $[1,2,3]$. Then,

$$
\forall d \in \Delta_{t, n, 3}: \operatorname{MSE}(d) \geq \operatorname{MSE}\left(d^{*}\right)
$$

Proof In the circular model, sequences $s$ from any of the three classes $B_{1}, B_{2}$ and $B_{3}$ all have the same $q_{11}(s)$ and $q_{12}(s)$, respectively. Assume the design $d$ has proportion $\pi_{2}$ of its sequences from one of the classes $B_{j}$ and $\pi_{3}$ from class $C$. Then, the proportion of sequences from class $A$ is $\pi_{1}=1-\pi_{2}-\pi_{3}$. We get from Table 3 that $q_{d 11}=$ $\sum \pi_{d}(s) q_{11}(s)=2\left(\frac{2}{3} \pi_{2}+\pi_{3}\right)$ and $q_{d 12}=-\left(\frac{2}{3} \pi_{2}+\pi_{3}\right)$. Hence,

$$
\begin{aligned}
\operatorname{MSE}(d) & \geq \frac{(t-1)^{2}}{n\left(\frac{2}{3} \pi_{2}+\pi_{3}\right)}+\delta\left(\frac{-\left(\frac{2}{3} \pi_{2}+\pi_{3}\right)}{2\left(\frac{2}{3} \pi_{2}+\pi_{3}\right)}\right)^{2} \\
& =\frac{(t-1)^{2}}{2 n\left(\frac{2}{3} \pi_{2}+\pi_{3}\right)}+\frac{\delta}{4} \geq \frac{(t-1)^{2}}{2 n}+\frac{\delta}{4}=\operatorname{MSE}\left(d^{*}\right)
\end{aligned}
$$

and $d^{*}$ is MSE-optimal. 
Table 4 Size of $q_{11}(s)$ and $q_{12}(s)$ for two classes of sequences of interest when $4 \leq p \leq t$

\begin{tabular}{llll}
\hline Class & Sequence & $q_{11}(s)$ & $q_{12}(s)$ \\
\hline$A$ & {$[1,2, \ldots, p-4, p-3, p-3, p-2, p-2]$} & $p-1-4 / p$ & $(p-4) / p$ \\
$B$ & {$[1, \ldots, p]$} & $p-1$ & -1 \\
\hline
\end{tabular}

Now consider the situation when $4 \leq p \leq t$. Remember that for a sequence $s$, the number $B_{s}$ denotes the number of periods in $s$ where the treatment of the preceding period is repeated. As in [12], we can show that

$$
\sum_{m=1}^{t} f_{s, m}^{2} \geq p+2 B_{s}
$$

also in the circular case. This inequality is used in the next proposition.

Proposition 1 Define $B(d)$ of a design $d \in \Delta_{t, n, p}$ as the weighted mean of the $B_{s}$ of its sequences, that is $B(d)=\sum_{s} B_{s} \pi_{d}(s)$. Then, for any $d \in \Delta_{t, n, p}$ we have

$$
\begin{aligned}
& q_{d 11} \leq p-1-\frac{2}{p} B(d) \\
& q_{d 12} \leq-1+\frac{p-2}{p} B(d) .
\end{aligned}
$$

Proof From the inequality $\sum_{m=1}^{t} f_{s, m}^{2} \geq p+2 B_{s}$, we get for any sequence $s$ that

$$
\begin{aligned}
& q_{11}(s) \leq p-1-\frac{2 B_{s}}{p} \\
& q_{12}(s) \leq B_{s}-1-\frac{2 B_{s}}{p}=-1+\frac{p-2}{p} B_{s} .
\end{aligned}
$$

Since $q_{d i j}=\sum \pi_{d}(s) q_{i j}(s)$, the desired inequalities follow.

For $4 \leq p \leq t$, there are two classes of sequences of interest. Class $A$ has representative sequence $[1,2, \ldots, p-4, p-3, p-3, p-2, p-2]$, i.e. two treatments are given twice in adjacent periods such that $B_{s}=2$. If $p>4$, there are $p-4$ other treatments given exactly once. The second class, $B$, has representative sequence $[1, \ldots, p]$, i.e. no treatment is given twice and $B_{s}=0$. Class $B$ has been shown to produce (universally) optimal designs for various cases $[2,5,8]$, since balanced designs use sequences from this class. Class $A$ was shown by Zheng et al. [13] to be important for non-trivial covariance matrices.

The respective values for $q_{11}(s)$ and $q_{12}(s)$ can be seen in Table 4 . We now show that a design minimizing the MSE can be found in the set of all designs consisting of sequences from classes $A$ and $B$ only. 
Proposition 2 Assume $4 \leq p \leq t$. For any design $d \in \Delta_{t, n, p}$ define

$$
\bar{\pi}=\frac{1}{2} \min \left\{B(d), \frac{p}{p-2}\right\}
$$

and

$$
M(\bar{\pi})=\frac{(t-1)^{2}}{n\left(p-1-\frac{4 \bar{\pi}}{p}\right)}+\delta\left(\frac{-1+2 \bar{\pi} \frac{p-2}{p}}{p-1-\frac{4 \bar{\pi}}{p}}\right)^{2} .
$$

We construct a symmetric design $\bar{d}_{t, n, p} \in \Delta_{t, n, p}$ which consists of a proportion $\bar{\pi}$ of sequences from class $A$ and a proportion $1-\bar{\pi}$ of sequences from class $B$. Then,

$$
\operatorname{MSE}(d) \geq M(\bar{\pi})=\operatorname{MSE}(\bar{d}) .
$$

Proof For the design $\bar{d}$, we conclude from the $q_{i j}(s)$ in Table 4 , that $q_{\bar{d} 11}=p-1-\frac{4}{p} \bar{\pi}$ and $q_{\bar{d} 12}=-(1-\bar{\pi})+\bar{\pi} \frac{p-4}{p}=-1+2 \bar{\pi} \frac{p-2}{p}$. Making use of the fact that $\bar{d}$ is symmetric, we then have

$$
\operatorname{MSE}(\bar{d})=\frac{(t-1)^{2}}{n q_{\bar{d} 11}}+\delta\left(\frac{q_{\bar{d} 12}}{q_{\bar{d} 11}}\right)^{2}=M(\bar{\tau}) .
$$

Now distinguish between two cases.

Case 1: $B(d) \geq \frac{p}{p-2}$.

In this case, $\bar{\pi}=\frac{p}{2(p-2)}$ and therefore $q_{\bar{d} 11}=p-1-\frac{2}{p} B(d)$ while $q_{\bar{d} 12}=0$. It follows from Proposition 1 that $q_{d 11} \leq p-1-\frac{2}{p} B(d) \leq q_{\bar{d} 11}$. Hence,

$$
M(\bar{\pi})=\operatorname{MSE}(\bar{d})=\frac{(t-1)^{2}}{n q_{\bar{d} 11}}+\delta\left(\frac{q_{\bar{d} 12}}{q_{\bar{d} 11}}\right)^{2}=\frac{(t-1)^{2}}{n q_{\bar{d} 11}} \leq \frac{(t-1)^{2}}{n q_{d 11}} \leq \operatorname{MSE}(d)
$$

Case 2: Assume $0 \leq B(d)<\frac{p}{p-2}$.

In that case, it follows from Proposition 1 that $q_{d 12} \leq-1+\frac{p-2}{p} B(d)<0$. Therefore, a lower bound for $\operatorname{MSE}(d)$ is achieved by making both $q_{d 12}$ and $q_{d 11}$ as large as possible. We hence get from Proposition 1 that

$$
\operatorname{MSE}(d) \geq \frac{(t-1)^{2}}{n\left(p-1-\frac{2 B(d)}{p}\right)}+\delta\left(\frac{-1+\frac{p-2}{p} B(d)}{p-1-\frac{2 B(d)}{p}}\right)^{2} .
$$

Since $\bar{\pi}=B(d) / 2$, the right hand side of this inequality equals $M(\bar{\pi})$.

It follows from Proposition 2 that the MSE-optimal design for a given $\delta$ can be found among the set of all designs $\bar{d}$ defined in Proposition 2 . That is, we have to find the best $\bar{\pi}$.

Theorem 5 Assume $4 \leq p \leq t$ and $\delta \geq 0$ is given. 
1. If $\delta<\frac{(p-1)(t-1)^{2}}{n p(p-3)}$ choose $d^{*}$ as the symmetric design that only consists of sequences from class B, i.e. a design $\bar{d}$ with $\bar{\pi}=0$. Then,

$$
\operatorname{MSE}\left(d^{*}\right)=M(0)=\min _{d \in \Delta_{t, n, p}} \operatorname{MSE}(d)
$$

2. If $\delta \geq \frac{(p-1)(t-1)^{2}}{n p(p-3)}$, let $d^{*}$ be a symmetric design that consists of sequences from class $A$ with proportion

$$
\pi(\delta)=\frac{p(p-1)}{4}-\frac{\delta n p^{3}(p-3)^{2}}{4\left(\delta n p(p-2)(p-3)-2(t-1)^{2}\right)}
$$

and of sequences from class $B$ with proportion $1-\pi(\delta)$. Then,

$$
\operatorname{MSE}\left(d^{*}\right)=M(\pi(\delta))=\min _{d \in \Delta_{t, n, p}} \operatorname{MSE}(d) .
$$

Proof It follows from Proposition 2 that for any $d$ there is a $\pi \in\left[0, \frac{p}{2(p-2)}\right]$ such that $\operatorname{MSE}(d) \geq M(\pi)$.

We therefore have to find a $\pi^{*} \in\left[0, \frac{p}{2(p-2)}\right]$ which minimizes $M(\pi)$.

Case 1: $\delta=\frac{2(t-1)^{2}}{n p(p-2)(p-3)}$. In this case the derivative of $M(\pi)$ with respect to $\pi$ is

$$
D(\pi)=\frac{4(t-1)^{2}(p-3)}{n(p-2)\left(p-1-\frac{4 \pi}{p}\right)^{3}}
$$

Then, $D(\pi)>0$ for all $\pi \in\left[0, \frac{p}{2(p-1)}\right]$ and $M(\pi)$ is minimal for $\pi=0$.

For all other $\delta$, the derivative of $M(\pi)$ with respect to $\pi$ is

$$
D(\pi)=\frac{8\left(\delta n p(p-2)(p-3)-2(t-1)^{2}\right)}{n p^{2}\left(p-1-\frac{4 \pi}{p}\right)^{3}}\left(\pi-\pi_{0}\right),
$$

where $\pi_{0}=\frac{p(p-1)}{4}-\frac{\delta n p^{3}(p-3)^{2}}{4\left(\delta n p(p-2)(p-3)-2(t-1)^{2}\right)}$.

Hence, $D(\pi)=0$ if and only if $\pi=\pi_{0}$.

Case $2: 0 \leq \delta<\frac{2(t-1)^{2}}{n p(p-2)(p-3)}$.

In this case, $\delta n p(p-2)(p-3)-2(t-1)^{2}<0$ and, therefore, $\pi_{0}>\frac{p(p-1)}{4}>$ $\frac{p}{2(p-1)}$. Further, it follows for all $\pi<\pi_{0}$ that $D(\pi)>0$. Hence, the minimum of $M(\pi)$ is attained for $\pi=0$.

Case 3: $\frac{2(t-1)^{2}}{n p(p-2)(p-3)}<\delta \leq \frac{(p-1)(t-1)^{2}}{n p(p-3)}$.

In this case, $\delta n p(p-2)(p-3)-2(t-1)^{2}>0$ and, therefore, $D(\pi)>0$ for all $\pi>\pi_{0}$. Also in this case, $\pi_{0} \leq 0$. Hence, again, the minimum of $M S E(\pi)$ is attained for $\pi=0$.

Combining cases 1,2 and 3, we have shown that for all $\delta \leq \frac{(p-1)(t-1)^{2}}{n p(p-3)}$ the choice $\pi^{*}=0$ is optimum. 
Case 4: $\delta \geq \frac{(p-1)(t-1)^{2}}{n p(p-3)}$. In this case, $\delta n p(p-2)(p-3)-2(t-1)^{2}>0$ and, therefore, $D(\pi)>0$ for all $\pi>\pi_{0}$ and $D(\pi)<0$ for all $\pi<\pi_{0}$. Therefore, the MSE is minimized by that $\bar{d}$ for which $\bar{\pi}=\pi_{0}=\pi(\delta)$.

Note that $\pi(\delta)$ increases with $\delta$ and approaches $\frac{p}{2(p-2)}$ for $\delta \rightarrow \infty$.

\section{Optimal Designs for the Model with Period Effects}

We extend the model and include period effects, i.e. the model becomes

$$
y=U \alpha+P \beta+T_{d} \tau+F_{d} \rho+\varepsilon
$$

with $\beta$ the vector of the period effects and $P$ the corresponding design matrix. In this model, we have the information matrices

$$
\begin{aligned}
\tilde{M}_{d 11} & =T_{d}^{T} \omega^{\perp}([U, P]) T_{d}, \\
\tilde{M}_{d 12} & =F_{d}^{T} \omega^{\perp}([U, P]) T_{d}, \\
\tilde{M}_{d 22} & =F_{d}^{T} \omega^{\perp}([U, P]) F_{d} .
\end{aligned}
$$

The MSE of a design then can be defined in the same manner as in the model without period effects, only replacing $M_{d i j}$ by $\tilde{M}_{d i j}, 1 \leq i \leq j \leq 2$. We get

$$
\widetilde{\operatorname{MSE}}(d)=\sigma^{2} \operatorname{tr}\left(\tilde{M}_{d 11}\right)^{+}+\delta \lambda_{1}\left(\tilde{M}_{d 12}^{T} \tilde{M}_{d 11}^{+} \tilde{M}_{d 11}^{+} \tilde{M}_{d 12}\right)
$$

Defining $\tilde{q}_{d 11}=\frac{1}{n} \operatorname{tr}\left(\tilde{M}_{d 11}\right)$ and $\tilde{q}_{d 12}=\frac{1}{n} \operatorname{tr}\left(\tilde{M}_{d 12}\right)$, we can show with the same arguments as before that

$$
\widetilde{\operatorname{MSE}}(d) \geq \frac{(t-1)^{2}}{n \tilde{q}_{d 11}}+\delta\left(\frac{\tilde{q}_{d 12}}{\tilde{q}_{d 11}}\right)^{2}
$$

with equality if $\tilde{M}_{d 11}$ and $\tilde{M}_{d 12}$ are completely symmetric.

Unfortunately, the calculations of $\operatorname{tr}\left(\tilde{M}_{d 11}\right)$ and $\operatorname{tr}\left(\tilde{M}_{d 12}\right)$ are different from the calculations before. In particular, it is not possible to express the $\tilde{q}_{d i j}$ as weighted means of contributions from the single sequences. Adapting the results of [4] to circular designs, we get

$$
\begin{aligned}
& \operatorname{tr}\left(\tilde{M}_{d 11}\right)=n \tilde{q}_{d 11}=n q_{d 11}-\frac{1}{n} \sum_{i=1}^{t} \sum_{k=1}^{p} l_{d i, k}^{2}+\frac{1}{n p} \sum_{i=1}^{t} r_{d i}^{2} \text { and } \\
& \operatorname{tr}\left(\tilde{M}_{d 12}\right)=n \tilde{q}_{d 12}=n q_{d 12}-\frac{1}{n} \sum_{i=1}^{t} \sum_{k=1}^{p} l_{d i, k} l_{d i, k-1}+\frac{1}{n p} \sum_{i=1}^{t} r_{d i}^{2}
\end{aligned}
$$


where $l_{d i, k}$ is the number of appearances of treatment $i$ in period $k$ (with $l_{d i 0}=l_{\mathrm{dip}}$ ) and $r_{d i}$ the number of appearances of treatment $i$ in the design. The numbers $q_{d 11}$ and $q_{d 12}$ are as in Sect. 3.

For symmetric designs, we get $\operatorname{tr}\left(\tilde{M}_{d 11}\right)=n q_{d 11}$ and $\operatorname{tr}\left(\tilde{M}_{d 12}\right)=n q_{d 12}$, i.e. $\tilde{q}_{d i j}=q_{d i j}$. In the case $p>t$, the best symmetric designs have $q_{d 12}=0$ and the results of Theorem 2 extend to the model with period effects.

There are examples-at least in non-circular designs—of designs that are nonsymmetric and where the uncorrected estimate from the model with period effects has a smaller bias than the estimate from the model without period effects. In the case $p \leq t$, the best symmetric designs have nonzero bias. Hence, it is not obvious that the results of Theorems 3 and 4 and of Proposition 2 still hold in the model with period effects.

To show that they do, we start by showing that for non-symmetric designs the loss in $\operatorname{tr}\left(\tilde{M}_{d 11}\right)$ is higher than the possible gain in $\left|\operatorname{tr}\left(\tilde{M}_{d 12}\right)\right|$.

Proposition 3 For any $d \in \Omega_{t, n, p}$, it holds that

$$
q_{d 11}-\tilde{q}_{d 11} \geq\left|q_{d 12}-\tilde{q}_{d 12}\right| .
$$

Proof Defining $Q=\frac{1}{n} P P^{T}-\frac{1}{n p} 1_{n p} 1_{n p}^{T}=\omega\left(\omega^{\perp}(U) P\right)$, we find that

$$
n q_{d 11}-n \tilde{q}_{d 11}=\frac{1}{n} \sum_{i=1}^{t} \sum_{k=1}^{p} l_{d i, k}^{2}-\frac{1}{n p} \sum_{i=1}^{t} r_{d i}^{2}=\operatorname{tr}\left(T_{d}^{T} Q T_{d}\right)
$$

and that

$$
n q_{d 12}-n \tilde{q}_{d 12}=\frac{1}{n} \sum_{i=1}^{t} \sum_{k=1}^{p} l_{d i, k} l_{d i, k-1}-\frac{1}{n p} \sum_{i=1}^{t} r_{d i}^{2}=\operatorname{tr}\left(T_{d}^{T} Q F_{d}\right) .
$$

Because of the circular structure of the carryover effects, we have

$$
T_{d}^{T} \omega^{\perp}([U, P]) T_{d}=F_{d}^{T} \omega^{\perp}([U, P]) F_{d}
$$

(cf. [8] or [6]) and, hence, that $T_{d}^{T} Q T_{d}=F_{d}^{T} Q F_{d}$. Therefore, the Cauchy-Schwarz inequality implies that

$$
\left|\operatorname{tr}\left(T_{d}^{T} Q F_{d}\right)\right| \leq \sqrt{\operatorname{tr}\left(T_{d}^{T} Q T_{d}\right) \operatorname{tr}\left(F_{d}^{T} Q F_{d}\right)}=\operatorname{tr}\left(T_{d}^{T} Q T_{d}\right)
$$

and the proposition follows.

If $p \geq 4$, we are now able to show that for every design $d$ there is a symmetric design $d_{s}$ consisting of sequences from classes $A$ and $B$ described in Table 4 only and having smaller or equal MSE. 
Proposition 4 Let $p \geq 4$. Assume the model with period effects holds and define $\Gamma_{t, n, p} \subset \Delta_{t, n, p}$ as the set of all symmetric $d \in \Delta_{t, n, p}$.

Then, $\forall d \in \Delta_{t, n, p} \exists d_{s} \in \Gamma_{t, n, p}$ with

$$
\widetilde{M S E}(d) \geq \widetilde{M S E}\left(d_{S}\right) .
$$

Proof Case 1: $\left|\tilde{q}_{d 12}\right|>1$.

Let $d_{s}$ be a symmetric design that consists of sequences from class $B$ only. Then, $d_{s} \in \Gamma_{t, n, p}$ and $\left|q_{d_{s} 12}\right|=1<\left|\tilde{q}_{d 12}\right|$. On the other hand (because of Proposition 1 and since $B\left(d_{S}\right)=0$ ), we have that

$$
q_{d_{s} 11} \geq q_{d 11} \geq \tilde{q}_{d 11}
$$

Thus, it holds that

$$
\widetilde{\operatorname{MSE}}(d) \geq \frac{(t-1)^{2}}{n q_{d 11}}+\delta\left(\frac{\tilde{q}_{d 12}}{q_{d 11}}\right)^{2} \geq \frac{(t-1)^{2}}{n q_{d_{s} 11}}+\delta\left(\frac{1}{q_{d_{s} 11}}\right)^{2}=\widetilde{\operatorname{MSE}}\left(d_{s}\right)
$$

Case 2: $\left|\tilde{q}_{d 12}\right| \leq 1$.

Let $d_{s}$ be the symmetric design that consists of sequences from class $A$ with proportion

$$
\pi=\frac{p\left(1-\left|\tilde{q}_{d 12}\right|\right)}{2(p-2)}
$$

and sequences from class $B$ with proportion $1-\pi$. Then, $d_{s} \in \Gamma_{t, n, p}$ and we get from Table 4 that

$$
q_{d_{s} 12}=-(1-\pi)+\pi \frac{p-4}{p}=-1+2 \pi \frac{p-2}{p}=-\left|\tilde{q}_{d 12}\right|
$$

(and hence $\left|q_{d_{s} 12}\right|=\left|\tilde{q}_{d 12}\right|$ ).

Therefore, if we can show that $\tilde{q}_{d_{s} 11} \geq \tilde{q}_{d 11}$, we are done.

Remembering that $d_{s}$ is a symmetric design we have $\tilde{q}_{d_{s} 11}=q_{d_{s} 11}$. Making use of Table 4 again, we get that $B\left(d_{S}\right)=2 \pi$ and

$$
\tilde{q}_{d_{s} 11}=\left(1-\frac{B\left(d_{s}\right)}{2}\right)(p-1)+\frac{B\left(d_{s}\right)}{2}\left(p-1-\frac{4}{p}\right)=p-1-\frac{2 B\left(d_{s}\right)}{p} .
$$

Subcase 2a: $B(d) \geq B\left(d_{s}\right)$.

From Proposition 1, we know that $q_{d 11} \leq p-1-\frac{2 B(d)}{p}$. Hence,

$$
\tilde{q}_{d 11} \leq q_{d 11} \leq p-1-\frac{2 B(d)}{p} \leq p-1-\frac{2 B\left(d_{s}\right)}{p}=\tilde{q}_{d_{s} 11} .
$$

Subcase 2b: $B(d)<B\left(d_{s}\right)$. 
Observe that

$$
\begin{aligned}
\tilde{q}_{d 12}-q_{d 12} & \geq-\left|\tilde{q}_{d 12}\right|-q_{d 12} \geq q_{d_{s} 12}+1-\frac{p-2}{p} B(d) \\
& =-1+\frac{p-2}{p} B\left(d_{s}\right)+1-\frac{p-2}{p} B(d)=\frac{p-2}{p}\left(B\left(d_{s}\right)-B(d)\right) \geq 0 .
\end{aligned}
$$

Hence, we conclude from Proposition 3 that

$$
q_{d 11}-\tilde{q}_{d 11} \geq \tilde{q}_{d 12}-q_{d 12} \geq \frac{p-2}{p}\left(B\left(d_{s}\right)-B(d)\right) .
$$

Therefore,

$$
\tilde{q}_{d 11} \leq q_{d 11}-\frac{p-2}{p}\left(B\left(d_{s}\right)-B(d)\right) .
$$

With Proposition 1, it follows that

$$
\tilde{q}_{d 11} \leq p-1-\frac{2 B(d)}{p}-\frac{p-2}{p}\left(B\left(d_{s}\right)-B(d)\right) .
$$

Since $p-2 \geq 2$, we get

$$
\tilde{q}_{d 11} \leq p-1-\frac{2 B\left(d_{s}\right)}{p}
$$

which is $\tilde{q}_{d_{s} 11}$. This completes the proof.

Note that this proposition implies that the design $d^{*}$ determined in Theorem 5 for the model without period effects, is also MSE-optimal in the model with period effects.

The proofs for the cases $p=2$ and $p=3$ have to be done differently. We start with $p=2$.

Theorem 6 Assume $2=p \leq t$. As in Theorem 3, consider the symmetric approximate design $d^{*} \in \Delta_{t, n, 2}$ consisting only of sequences from class $B$ of Table 2 with representative sequence $[1,2]$. We then have $\forall d \in \Omega_{t, n, 2}$ that

$$
\widetilde{M S E}(d) \geq \widetilde{M S E}\left(d^{*}\right)
$$

Proof We have seen in the proof of Theorem 3 for an arbitrary design $d$ that

$$
q_{d 11}=\pi_{2} \text { and } q_{d 12}=-\pi_{2}
$$

where $\pi_{2}$ is the proportion of sequences from class $B$.

In the model with period effects, we write $\tilde{q}_{d 11}=q_{d 11}-\epsilon=\pi_{2}-\epsilon$. Since $q_{d 11} \geq \tilde{q}_{d 11} \geq 0$, we have $\pi_{2} \geq \epsilon \geq 0$. 
From Proposition 3, we get that $\left|q_{d 12}-\tilde{q}_{d 12}\right| \leq \epsilon$. Therefore, $\tilde{q}_{d 12} \leq q_{d 12}+\epsilon=$ $-\pi_{2}+\epsilon \leq 0$ and $\left|\tilde{q}_{d 12}\right| \geq \pi_{2}-\epsilon$.

It follows that

$$
\widetilde{\operatorname{MSE}}(d) \geq \frac{(t-1)^{2}}{n \pi_{2}-\epsilon}+\delta\left(\frac{\pi_{2}-\epsilon}{\pi_{2}-\epsilon}\right)^{2} \geq \frac{(t-1)^{2}}{n}+\delta=\widetilde{\operatorname{MSE}}\left(d_{B}\right) .
$$

This completes the proof.

The proof for the case $p=3$ turns out to cause extra difficulties.

Theorem 7 Assume $3=p \leq t$. As in Theorem 4, consider a symmetric approximate design $d^{*} \in \Delta_{t, n, 3}$ that consists only of sequences from class $C$ with representative sequence $[1,2,3]$. Then,

$$
\forall d \in \Delta_{t, n, 3}: \widetilde{M S E}(d) \geq \widetilde{M S E}\left(d^{*}\right) .
$$

Proof We have seen in the proof of Theorem 4 for an arbitrary design $d \in \Delta_{t, n, 3}$ that

$$
q_{d 11}=2\left(\pi_{3}+\frac{2}{3} \pi_{2}\right) \text { and } q_{d 12}=-\left(\pi_{3}-\frac{2}{3} \pi_{2}\right) .
$$

If we define $\epsilon=q_{d 11}-\tilde{q}_{d 11}$ and $\rho=\tilde{q}_{d 12}-q_{d 12}$, we get that

$$
\widetilde{\operatorname{MSE}}(d) \geq \frac{(t-1)^{2}}{n\left(2\left(\pi_{3}+\frac{2}{3} \pi_{2}\right)-\epsilon\right)}+\delta\left(\frac{\pi_{3}+\frac{2}{3} \pi_{2}-\rho}{2\left(\pi_{3}+\frac{2}{3} \pi_{2}\right)-\epsilon}\right)^{2} .
$$

At this point, Proposition 3 is of no use. It only guarantees that $\epsilon \geq|\rho|$, but if we could find a design with $\rho=\epsilon>0$, this would produce a smaller bias. If we could get $\rho=\epsilon=\pi_{3}+\frac{2}{3} \pi_{2}$, we could even achieve

$$
\left(\frac{\pi_{3}+\frac{2}{3} \pi_{2}-\rho}{2\left(\pi_{3}+\frac{2}{3} \pi_{2}\right)-\epsilon}\right)^{2}=0
$$

However, this is not possible. In the case $p=3$, we can get a stronger version of Proposition 3.

In the proof of Proposition 3, we have used the equalities

$$
n q_{d 11}-n \tilde{q}_{d 11}=\frac{1}{n} \sum_{i=1}^{t} \sum_{k=1}^{p} l_{d i k}^{2}-\frac{1}{n p} \sum_{i=1}^{t} r_{d i}^{2}
$$

and

$$
n q_{d 12}-n \tilde{q}_{d 12}=\frac{1}{n} \sum_{i=1}^{t} \sum_{k=1}^{p} l_{d i, k-1} l_{d i k}-\frac{1}{n p} \sum_{i=1}^{t} r_{d i}^{2}
$$


Hence,

$$
\begin{aligned}
\epsilon & =\frac{1}{n^{2}} \sum_{i=1}^{t}\left(\sum_{k=1}^{3} l_{d i, k}^{2}-\frac{1}{3}\left(\sum_{k=1}^{3} l_{d i, k}\right)^{2}\right) \\
& =\frac{1}{n^{2}} \sum_{i=1}^{t} \frac{2}{3}\left(l_{d i, 1}^{2}+l_{d i, 2}^{2}+l_{d i, 3}^{2}-l_{d i, 1} l_{d i, 3}-l_{d i, 2} l_{d i, 1}-l_{d i, 3} l_{d i, 2}\right)
\end{aligned}
$$

and

$$
\begin{aligned}
\rho & =-\frac{1}{n^{2}} \sum_{i=1}^{t}\left(\sum_{k=1}^{3} l_{d i, k} l_{d i, k-1}-\frac{1}{3}\left(\sum_{k=1}^{3} l_{d i, k}\right)^{2}\right) \\
& =\frac{1}{n^{2}} \sum_{i=1}^{t} \frac{1}{3}\left(l_{d i, 1}^{2}+l_{d i, 2}^{2}+l_{d i, 3}^{2}-l_{d i, 1} l_{d i, 3}-l_{d i, 2} l_{d i, 1}-l_{d i, 3} l_{d i, 2}\right) .
\end{aligned}
$$

Therefore, $\rho=\frac{1}{2} \epsilon$, regardless of our choice of the $l_{d i, k}$. It follows that

$$
\widetilde{\operatorname{MSE}}(d) \geq \frac{(t-1)^{2}}{n\left(2\left(\pi_{3}+\frac{2}{3} \pi_{2}\right)-\epsilon\right)}+\frac{\delta}{4} \geq \frac{(t-1)^{2}}{2 n}+\frac{\delta}{4}=\widetilde{\operatorname{MSE}}\left(d^{*}\right)
$$

\section{Efficiency in Terms of MSE and Example}

We have seen in Sects. 3 and 4 that MSE-optimality, for $4 \leq p \leq t$, is a local criterion, the MSE-optimum design depends on the true $\delta$. Since in practice, we will in general not know $\delta$, we try to find a design which is efficient for a broad range of $\delta$. The efficiency of a design $d$ at a point $\delta$ is defined as

$$
\operatorname{Eff}_{\delta}(d)=\operatorname{MSE}_{\delta, \mathrm{opt}} / \operatorname{MSE}_{\delta}(d)
$$

where $\mathrm{MSE}_{\delta \text {, opt }}$ is the minimum MSE for this $\delta$. Thus, a high efficiency is desirable and the efficiency is a number between 0 and 1 .

In what follows, we consider the case $4 \leq p \leq t$. In the case of circular designs, often designs are considered that only consist of sequences from class $B$. Special cases of these designs are so-called circular balanced designs or orthogonal arrays of type I. These have been shown to be optimal in a broad class of designs, see, e.g. [2]. However, Zheng et al. [13] showed that designs with sequences from class $A$ can be efficient in some situations. Thus, we will consider the designs $d_{A}$ and $d_{B}$ that only consist of sequences from classes $A$ or $B$, respectively, and calculate their efficiency. 


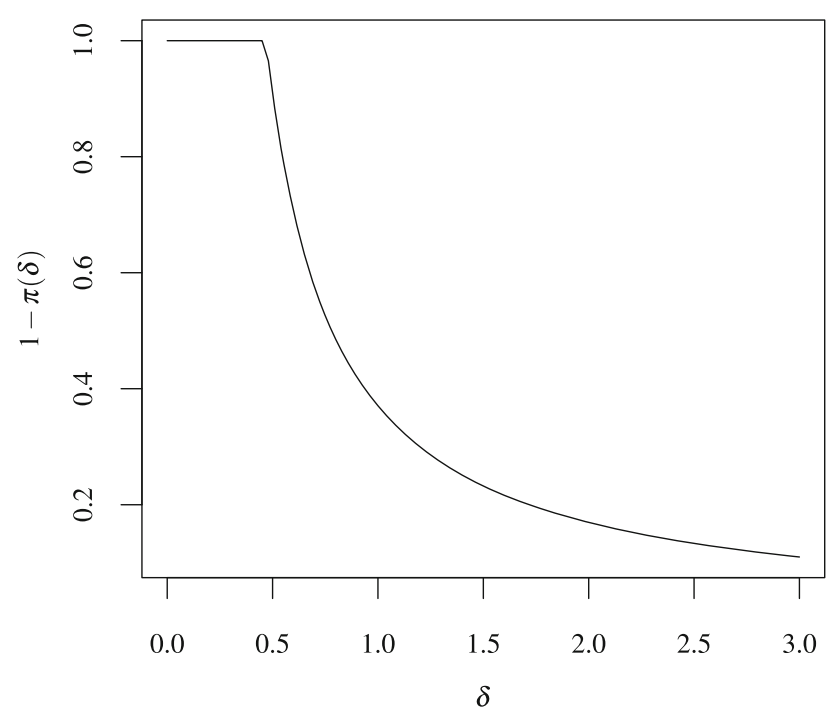

Fig. 1 Proportion $1-\pi(\delta)$ of sequences from class $B$ as a function of $\delta$

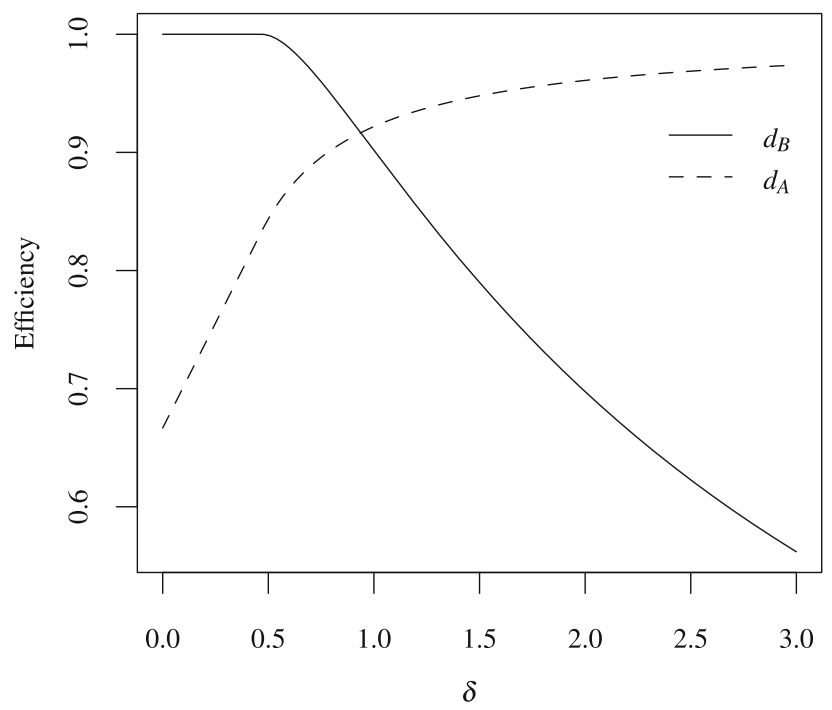

Fig. 2 MSE-efficiencies for designs $d_{B}$ (solid line) and $d_{A}$ (dashed line) for parameters $p=6, t=4, n=$ 40 and $\delta \in[0,3]$

First, we examine the case that $\delta<\frac{(p-1)(t-1)^{2}}{n p(p-3)}$. In this case, $d_{B}$ is MSE-optimal and thus

$$
\operatorname{Eff}_{\delta}\left(d_{B}\right)=1
$$


For $d_{A}$, we get in this case

$$
\operatorname{Eff}_{\delta}\left(d_{A}\right)=\frac{-\left(\delta n+(p-1)(t-1)^{2}\right)\left(p^{2}-p-4\right)^{2}}{(p-1)^{2}\left(4 p-t p(t-2)\left(p^{2}-p-4\right)+p^{2}(p-1)+\delta n(p-4)^{2}\right)} .
$$

If $\delta \geq \frac{(p-1)(t-1)^{2}}{n p(p-3)}$, we get

$$
\operatorname{Eff}_{\delta}\left(d_{B}\right)=\frac{\left(\delta n p\left(p^{2}-5 p+6\right)-(t-1)^{2}\right)(t-1)^{2}(p-1)^{2}}{\delta n p^{2}(p-3)^{2}\left(\delta n+(p-1)(t-1)^{2}\right)}
$$

and

$$
\operatorname{Eff}_{\delta}\left(d_{A}\right)=\frac{\left(\delta n p\left(p^{2}-5 p+6\right)-(t-1)^{2}\right)(t-1)^{2}\left(p^{2}-p-4\right)^{2}}{\delta n p^{2}(p-3)^{2}\left(p(t-1)^{2}\left(p^{2}-p-4\right)+n \delta(p-4)^{2}\right)}
$$

We see that the efficiency of $d_{B}$ decreases with $\delta$ while the efficiency of $d_{A}$ increases with $\delta$.

As an example, consider the case $t=6, p=4$ and $n=40$. In that case, the critical step for $\pi(\delta)$ is at $\delta_{1}=\frac{4 \cdot 25}{40 \cdot 4 \cdot 1}=\frac{15}{32}=0.46875$. If $\delta<\delta_{1}$, the design $d_{B}$ is MSE-optimal and has MSE-efficiency 1.

If $\delta$ increases, we see that the proportion $1-\pi(\delta)$ of sequences from class $B$ in the MSE-optimal design decreases (Fig. 1). This is in agreement with the decreasing efficiency of the design $d_{B}$ and the increasing efficiency of the design that only consists of sequences from class $A$ (Fig. 2). However, as long as $\delta$ remains reasonably small, the efficiency of $d_{B}$ is still high (and larger than the efficiency of $d_{A}$ ). Therefore, it makes sense to use the design $d_{B}$.

Of particular interest for practice is the case when the number of treatments $t$ equals the number of periods $p$. In this case, we can construct a circular balanced uniform design $d_{B}$ with $n=t(t-1)$ units.

For a design of this size, the limit $\delta_{1}=\frac{(p-1)(t-1)^{2}}{n p(p-3)}$ becomes 0.56 for $p=4,0.32$ for $p=5,0.23$ for $p=6$ and goes down to 0.01 for $p=100$. The efficiency $\operatorname{Eff}_{d_{B}}(\delta)$ is 1 as long as $\delta$ is less than $\delta_{1}$, and decreases when $\delta$ increases. For $\delta=3$, we observe that $\operatorname{Eff}_{d_{B}}(3)$ is 0.62 if $p=4,0.61$ if $p=5$ and 0.64 if $p=6$. For larger $p$, it increases, for instance, it is 0.97 for $p=100$.

Funding This research was sponsored by the Deutsche Forschungsgemeinschaft (SFB 823, Teilprojekt C2).

\section{Declarations}

Conflict of interest The authors declare that there are no conflicts of interest.

Open Access This article is licensed under a Creative Commons Attribution 4.0 International License, which permits use, sharing, adaptation, distribution and reproduction in any medium or format, as long as you give appropriate credit to the original author(s) and the source, provide a link to the Creative Commons licence, and indicate if changes were made. The images or other third party material in this article are included in the article's Creative Commons licence, unless indicated otherwise in a credit line to the material. If material is not included in the article's Creative Commons licence and your intended use is not permitted 
by statutory regulation or exceeds the permitted use, you will need to obtain permission directly from the copyright holder. To view a copy of this licence, visit http://creativecommons.org/licenses/by/4.0/.

\section{References}

1. Azaïs JM (1987) Design of experiments for studying intergenotypic competition. J R Stat Soc B 49:334-345

2. Bailey RA, Cameron PJ, Filipiak K, Kunert J, Markiewicz A (2017) On optimality and construction of circular repeated-measurements designs. Stat Sinica 27:1-22

3. Bose M, Dey A (2009) Optimal crossover designs. World Scientific, Hackensack, NJ

4. Cheng CS, Wu CF (1980) Balanced repeated measurements designs. Ann Stat 8:1272-1283

5. Druilhet P (1999) Optimality of neighbour balanced designs. J Stat Plann Inference 81:141-152

6. Druilhet P, Bailey RA (2004) Optimality of neighbor-balanced designs for total effects. Ann Stat 32(4):1650-1661. https://doi.org/10.1214/009053604000000481

7. Kunert J (1983) Optimal design and refinement of the linear model with applications to repeated measurements designs. Ann Stat 11:247-257

8. Kunert J (1998) On the analysis of circular balanced crossover designs. J Stat Plann Inference 69:359370

9. Kushner HB (1997) Optimal repeated measurements designs: the linear optimality equations. Ann Stat 25:2328-2344

10. Magda CG (1980) Circular balanced repeated measurements designs. Commun Stat Theory Methods 9:1901-1918

11. Naes T, Brockhoff P, Tomic O (2010) Statistics for sensory and consumer science. Wiley, Chichester

12. Neumann C, Kunert J (2018) On MSE-optimal crossover designs. Ann Stat 46:2939-2959

13. Zheng W, Ai M, Li K (2017) Identification of universally optimal circular designs for the interference model. Ann. Stat 45:1462-1487 